\title{
Solución local de una Ecuación de Kirchhoff no lineal viscoelástica con término disipativo
}

\author{
Teófanes Quispe Méndez ${ }^{1}$ \\ tquispem@unmsm.edu.pe
}

\begin{abstract}
Resumen
En este trabajo, estudiamos la existencia y unicidad de soluciones locales del problema mixto para un tipo de ecuación de Kirchhoff no lineal viscoelástica con término disipativo.

Palabras Clave: Solución Local, Ecuación de Kirchhoff no lineal viscoelástica, Método de Galerkin, Método del punto fijo.
\end{abstract}

\section{Abstract}

In this work, we study the existence and uniqueness of the local solutions to the mixed problem for a type of viscoelastic nonlinear Kirchhoff's equation with dissipative term.

Keywords: Local solution, Viscoelastic nonlinear Kirchhoff's equation, Galerkin method, Fixed point method.

\section{Introducción}

En este artículo consideramos el siguiente problema de valores iniciales y frontera

$$
\begin{array}{ll}
u_{t t}+\alpha(x, t) u_{t}-\beta(t) \Delta u_{t}-M\left(\int_{\Omega}|\nabla u|^{2} d x\right) \Delta u & \\
\qquad \int_{0}^{t} g(t-s) \Delta u(s) d s=f(u) & , x \in \Omega, t \geq 0, \\
u=0 & , x \in \partial \Omega, t \geq 0, \\
u(x, 0)=u_{0}(x), \quad u_{t}(x, 0)=u_{1}(x) & , x \in \Omega,
\end{array}
$$

\footnotetext{
${ }^{1}$ UNMSM, Facultad de Ciencias Matemáticas, Lima - Perú.
} 
donde $\Omega$ es un conjunto abierto y acotado de $\mathbb{R}^{n}$ con frontera bien regular $\partial \Omega$, $\nabla$ es el gradiente, $\Delta$ el operador laplaciano, $\alpha(x, t)$ es una función real para $x \in \Omega, t \geq 0, \beta(t)$ función real continua positiva para $t \geq 0, M(s)$ función real positiva para $s \geq 0, g(t)$ función real continua no negativa para $t \geq 0$ y $f(s)$ función real no lineal para $s \in \mathbb{R}$.

El caso $n=1$, el problema (1.1) describe las vibraciones transversales no lineales de una cuerda de material viscoelástico, fuertemente tensa entre dos puntos fijos $x=0$ y $x=L$, en el eje $x$ del plano $x u$. La ecuación resultante es

$$
\begin{aligned}
\rho h u_{t t}+\alpha(x, t) u_{t}-\beta(t) \Delta u_{t}-\left(p_{o}\right. & \left.+\frac{E h}{2 L} \int_{0}^{L}\left|u_{x}\right|^{2} d x\right) u_{x x} \\
& +\int_{0}^{t} g(t-s) u_{x x}(s) d s=f(u),
\end{aligned}
$$

donde $u=u(x, t)$ es el desplazamiento transversal en el espacio de coordenada $x$ y en el tiempo $t, \rho$ es la densidad de masa, $h$ el área de la sección transversal de la cuerda, $p_{0}$ es la tensión inicial, $E$ el módulo de Young del material, $\alpha(x, t)$ y $\beta(t)$ son los coeficientes de las fuerzas amortiguadoras, $g(t)$ la función de relajación, y $f(u)$ la fuerza restauradora. Cuando $\alpha \equiv \beta \equiv g \equiv 0$ y la cuerda de material elástica, la ecuación (1.2) fue propuesto y estudiado primero por Kirchhoff [6]. El caso general $n \geq 1$, la ecuación en $(1,1)$ tiene diversas aplicaciones, como en el área de óptica no lineal, física del plasma y mecánica de fluidos. Cuando $g$ es una función no trivial y $M \equiv 1$, la ecuación en (1.1) fue investigada desde diferentes puntos de vista por diversos autores y la referencia citado por ellos $[2,3,5,8,9]$. Para $\beta \equiv 0$ y $\alpha(x, t)=a(x)$ no nulo en una parte del dominio $\Omega$, Cavalcanti et al. [5], obtuvieron el decaimiento exponencial de las soluciones. Para $\alpha \equiv \beta \equiv 0$ y $f(u)=|u|^{\gamma} u$ con $\gamma>0$, Berrimi and Messaoudi [3] obtuvieron la existencia y el decaimiento exponencial de las soluciones, y Wu [9] obtiene la singularidad de las soluciones. Cuando $g$ es una función no trivial, $M$ es una función no constante, $\alpha \equiv 0$ y $\beta \equiv 1$, recientemente, Wu and Tsai [10] estudiaron la existencia, el decaimiento exponencial y la singularidad de las soluciones de la ecuación en $(1,1)$.

En este trabajo probaremos la existencia y la unicidad de soluciones locales del problema (1.1), cuando $g$ es una función continua no negatina, $M$ función continua positiva, $\alpha$ función de un espacio determinado, $\beta$ función continua positiva y $f$ función real no lineal. Primero probaremos la existencia y unicidad de solución global de un problema lineal asociado a (1.1), utilizando el método de Galerkin. Asi mismo obtendremos una estimativa para sus soluciones. En segundo lugar, linealizaremos el problema (1.1) para elementos de un espacio $G_{T_{0}, R_{0}}$, llamado Espacio de soluciones, luego obtendremos sus soluciones en $G_{T_{0}, R_{0}}$ y después, por argumentos del teorema del punto fijo de Banach, tendremos las soluciones locales del problema (1.1) sobre un intervalo $\left[0, T_{0}\right]$, donde $T_{0}>0$ depende de los datos iniciales y los parámetros del problema. La prueba de unicidad de soluciones, la obtendremos utilizando las estimativas de soluciones de un problema lineal. En la discución del problema emplearemos las estrategias seguidas por Andrade and Mognon [1], Berrimi and Messaoudi [3], y Wu and Tsai [10]. 


\section{Preliminares}

En esta sección daremos algunas notaciones, conceptos y lemas sin demostración que serán utilizados en el desarrollo del presente trabajo.

Sea $\Omega$ un conjunto abierto y acotado de $\mathbb{R}^{n}$ con frontera bien regular $\partial \Omega$. Denotamos el producto interno y la norma de $L^{2}(\Omega)$ y $L^{p}(\Omega)$, con $(.,$.$) y |\cdot|_{p}$, respectivamente, para $1 \leq p \leq \infty$. Además $((.,)$.$) y \|$.$\| , denotarán el producto interno y la norma de H_{0}^{1}(\Omega)$, donde $((u, v)):=\int_{\Omega} \nabla u(x) . \nabla v(x) d x$ es la forma de Dirichlet.

Sea $X$ un espacio de Banach, $0<T \leq \infty$ y $1 \leq p \leq \infty$. Representamos con $L^{p}(0, T ; X)$ al espacio de Banach de las funciones vectoriales $u:] 0, T[\longrightarrow X$ tales que son medibles y $\|u(t)\|_{X} \in L^{p}(0, T)$, con la norma

$$
\begin{gathered}
\|u\|_{L^{p}(0, T ; X)}:=\left(\int_{0}^{T}\|u(t)\|_{X}^{p} d t\right)^{\frac{1}{p}}, 1 \leq p<\infty, \\
\|u\|_{L^{\infty}(0, T ; X)}:=\sup _{0<t<T} \operatorname{ess}\|u(t)\|_{X}, \quad p=\infty .
\end{gathered}
$$

Similarmente, cuando $0<T<\infty$, representaremos con $C([0, T] ; X)$ al espacio de Banach de las funciones continuas $u:[0, T] \longrightarrow X$, con la norma

$$
\|u\|_{C([0, T] ; X)}:=\sup _{0 \leq t \leq T}\|u(t)\|_{X} .
$$

Denotamos $v^{\prime}:=v_{t}, v^{\prime \prime}:=v_{t t}, v(t)(x):=v(x, t), L^{1}:=L^{1}(0, T)$ y $L^{\infty}:=L^{\infty}(\Omega)$.

Hipótesis. Imponemos sobre las funciones reales $\mu(x), \alpha(x, t), \beta(t), g(t), M(s)$ y $f(s)$ las siguientes condiciones:

(H1) $\mu \in C\left(\left[0, \infty[), \mu^{\prime} \in L^{2}(0, \infty)\right.\right.$ y $\mu(t) \geq m_{0}>0, \forall t \geq 0, m_{0}$ constante.

(H2) $\alpha \in L^{2}\left(0, \infty ; L^{\infty}(\Omega)\right)$.

(H3) $\beta \in C\left(\left[0, \infty[), \beta^{\prime} \in L^{1}(0, \infty)\right.\right.$ y $\beta(t) \geq \beta_{0}>0, \forall t \geq 0, \beta_{0}$ constante.

(H4) $g \in C^{1}([0, \infty[)$, acotada, $g(t) \geq 0, \forall t \geq 0$,

$$
m_{0}-\int_{0}^{\infty} g(s) d s:=l>0
$$

y existen constantes positivas $C_{1}$ y $C_{2}$ tal que

$$
-C_{1} g(t) \leq g^{\prime}(t) \leq-C_{2} g(t), \forall t \geq 0 .
$$

(H5) $M \in C^{1}\left(\left[0, \infty[)\right.\right.$ y $M(s) \geq m_{0}>0, \forall s \geq 0$. 
(H6) $f(0)=0$ y existe una constante positiva $K$ tal que

$$
|f(x)-f(y)| \leq K|x-y|\left(|x|^{p-2}+|y|^{p-2}\right), \forall x, y \in \mathbb{R},
$$

con $2 \leq p \leq \frac{2(n-1)}{n-2}$ para $n \geq 3$ o $p \geq 2$ para $n \leq 2$.

Lema 2.1 (Desigualdad de Sobolev-Poincaré [4]). Si $2 \leq p \leq \frac{2 n}{n-2}$ para $n \geq 3$ o $p \geq 2$ para $n \leq 2$, entonces existe una constante positiva $B_{1}$ tal que

$$
|u|_{p} \leq B_{1}\|u\|, \forall u \in H_{0}^{1}(\Omega)
$$

$y$

$$
\|u\| \leq B_{1}|\Delta u|_{2}, \forall u \in H_{0}^{1}(\Omega) \cap H^{2}(\Omega) .
$$

Lema 2.2 (Desigualdad de Yaung [4]). Sea $u \in L^{p}\left(\mathbb{R}^{m}\right)$ y $v \in L^{q}\left(\mathbb{R}^{m}\right)$ con $1 \leq p \leq \infty$, $1 \leq q \leq \infty$ y $r$ un número real verificando $\frac{1}{r}=\frac{1}{p}+\frac{1}{q}-1 \geq 0$. Entonces $u * v \in L^{r}\left(\mathbb{R}^{m}\right)$ y

$$
\|u * v\|_{L^{r}\left(\mathbb{R}^{m}\right)} \leq\|u\|_{L^{p}\left(\mathbb{R}^{m}\right)}\|v\|_{L^{q}\left(\mathbb{R}^{m}\right)},
$$

donde la convolución de $u$ con $v$ está definida por

$$
(u * v)(x):=\int_{\mathbb{R}^{m}} u(x-y) v(y) d y .
$$

Lema 2.3. Si $g \in C^{1}\left(\left[0, \infty[)\right.\right.$ y $w \in C^{1}\left([0, T] ; L^{2}(\Omega)\right)$, entonces

$$
\begin{aligned}
\int_{0}^{t} g(t-s)\left(w(s), w^{\prime}(t)\right) d s= & -\frac{1}{2} \frac{d}{d t}\left[(g \square w)(t)-\left(\int_{0}^{t} g(s) d s\right)|w(t)|_{2}^{2}\right] \\
& -\frac{1}{2} g(t)|w(t)|_{2}^{2}+\frac{1}{2}\left(g^{\prime} \square w\right)(t),
\end{aligned}
$$

donde

$$
(v \square w)(t):=\int_{0}^{t} v(t-s)|w(t)-w(s)|_{2}^{2} d s .
$$

Demostración. Diferenciando el término $g \square w$, se obtiene el resultado.

Lema 2.4 (Desigualdad de Gronwall [11]). Sean $f, g:[a, b] \rightarrow \mathbb{R}$ continuas, con $g$ no decreciente $y \in L^{1}(a, b)$, que satisfacen la desigualdad

$$
f(t) \leq g(t)+\int_{a}^{t} h(s) f(s) d s, \forall t \in[a, b] .
$$


Entonces,

$$
f(t) \leq g(t) \exp \left(\int_{a}^{t} h(s) d s\right), \forall t \in[a, b]
$$

Definición 2.5. Una función $u: \Omega \times[0, T] \rightarrow R$ es llamada solución del problema (1.1) sobre $[0, T]$ si satisface las condiciones $(1.1)_{2}-(1.1)_{3}$ y la igualdad

$$
\begin{aligned}
u_{t t}+\alpha(x, t) u_{t}-\beta(t) & \Delta u_{t}-M\left(\int_{\Omega}|\nabla u|^{2} d x\right) \Delta u \\
+ & \int_{0}^{t} g(t-s) \Delta u(s) d s=f(u) \text { en } L^{2}\left(0, T ; H^{-1}(\Omega)\right) .
\end{aligned}
$$

\section{Problema No Lineal}

En esta sección estudiaremos la solución global del siguiente problema lineal

$$
\begin{aligned}
& u_{t t}+\alpha(x, t) u_{t}-\beta(t) \Delta u_{t}-\mu(t) \Delta u \\
& \left.+\int_{0}^{t} g(t-s) \Delta u(s) d s=h(x, t) \text { en } \Omega \times\right] 0, T[\text {, } \\
& u=0 \\
& u(x, 0)=u_{0}(x), \quad u_{t}(x, 0)=u_{1}(x) \\
& \text { en } \partial \Omega \times] 0, T[\text {, } \\
& \text { en } \Omega \text {, }
\end{aligned}
$$

donde $T$ es un número real positivo arbitrario fijo.

Lema 3.1. Supongamos que las funciones $\mu, \alpha, \beta$ y g satisfacen las hipótesis (H1), (H2), (H3) y (H4), respectivamente, y que $u_{0} \in H_{0}^{1}(\Omega) \cap H^{2}(\Omega), u_{1} \in H_{0}^{1}(\Omega)$ y $h \in L^{2}\left(0, T ; L^{2}(\Omega)\right)$. Entonces el problema (3.1) admite una única solución u sobre $[0, T]$ tal que

$$
\begin{aligned}
& u \in L^{\infty}\left(0, T ; H_{0}^{1}(\Omega) \cap H^{2}(\Omega)\right), \\
& u^{\prime} \in L^{\infty}\left(0, T ; H_{0}^{1}(\Omega)\right) \cap L^{2}\left(0, T ; H_{0}^{1}(\Omega)\right), \\
& u^{\prime \prime} \in L^{2}\left(0, T ; L^{2}(\Omega)\right) .
\end{aligned}
$$

Además, la solución u verifica la siguiente estimativa

$$
E_{1}(t) \leq\left[E_{1}^{\frac{1}{2}}(0)+\int_{0}^{t}|h(s)|_{2} d s\right]^{2} \exp \left(\int_{0}^{t}\left[\frac{\left|\mu^{\prime}(s)\right|}{\mu_{g}(s)}+|\alpha(s)|_{L^{\infty}}\right] d s\right),
$$

$\forall t \in[0, T]$, donde

$$
\begin{aligned}
& E_{1}(t):=\frac{1}{2}\left|u^{\prime}(t)\right|_{2}^{2}+\frac{1}{2} \mu_{g}(t)\|u(t)\|^{2}+\frac{1}{2}(g \square \nabla u)(t)+\int_{0}^{t} \beta(s)\left\|u^{\prime}(s)\right\|^{2} d s, \\
& \mu_{g}(t):=\mu(t)-\int_{0}^{t} g(s) d s .
\end{aligned}
$$


Demostración. Procedemos por etapas.

Soluciones Aproximadas. Sea $\left\{\omega_{k}\right\}$ una base hilbertiana de $H_{0}^{1}(\Omega) \cap H^{2}(\Omega)$ formada por las funciones propias del operador elíptico $-\triangle$.

Sea $V_{m}=\left[\omega_{1}, \omega_{2}, \ldots, \omega_{m}\right]$ el subespacio generado por los primeros $m$ vectores $\omega_{1}, \omega_{2}, \ldots, \omega_{m}$ de $\left\{\omega_{k}\right\}$. Sea

$$
u_{m}(t)=\sum_{j=1}^{m} r_{j m}(t) \omega_{j},
$$

las soluciones aproximadas en $V_{m}$ del problema (3.1), donde las funciones $r_{j m}(t)$, $j=1,2, \ldots, m$, son determinadas del siguiente problema en ecuaciones diferenciales ordinarias, para $w \in V_{m}$

$$
\begin{gathered}
\left(u_{m}^{\prime \prime}(t), w\right)+\left(\alpha(t) u_{m}^{\prime}(t), w\right)+\beta(t)\left(\left(u_{m}^{\prime}, w\right)\right)+\mu(t)\left(\left(u_{m}(t), w\right)\right) \\
\quad-\int_{0}^{t} g(t-s)\left(\left(u_{m}(s), w\right)\right) d s=(h(t), w), \\
u_{m}(0)=u_{0 m}, \quad u_{m}^{\prime}(0)=u_{1 m},
\end{gathered}
$$

donde

$$
\begin{aligned}
& u_{0 m}=\sum_{j=1}^{m} r_{0 j m} w_{j}, \quad u_{0 m} \rightarrow u_{0} \quad \text { fuerte en } H_{0}^{1}(\Omega) \cap H^{2}(\Omega), \\
& u_{1 m}=\sum_{j=1}^{m} r_{1 j m} w_{j}, \quad u_{1 m} \rightarrow u_{1} \quad \text { fuerte en } H_{0}^{1}(\Omega) .
\end{aligned}
$$

Por el Teorema de Carathéodory[6], aseguramos la existencia de una solución local $u_{m}$ del problema aproximado (3.3) en el intervalo $\left[0, T_{m}[\right.$. Las siguientes estimativas a priori nos permitirán extender la solución $u_{m}$ al intervalo $[0, T]$ independiente de $m$.

Estimativa a Priori I. Tomando $w=u_{m}^{\prime}(t)$ en (3.3), obtenemos

$$
\begin{aligned}
& \frac{1}{2} \frac{d}{d t}\left|u_{m}^{\prime}(t)\right|_{2}^{2}+\left(\alpha(t) u_{m}^{\prime}(t), u_{m}^{\prime}(t)\right) \\
& +\beta(t)\left\|u_{m}^{\prime}(t)\right\|^{2}+\frac{1}{2} \mu(t) \frac{d}{d t}\left\|u_{m}(t)\right\|^{2} \\
& \quad-\int_{0}^{t} g(t-s)\left(\left(u_{m}(s), u_{m}^{\prime}(t)\right)\right) d s=\left(h(t), u_{m}^{\prime}(t)\right) .
\end{aligned}
$$

Del Lema 2.3, resulta

$$
\begin{aligned}
-\int_{0}^{t} g(t-s)\left(\left(u_{m}(s), u_{m}^{\prime}(t)\right)\right) d s= & \frac{1}{2} \frac{d}{d t}\left[\left(g \square \nabla u_{m}\right)(t)\right. \\
& \left.\left.-\left(\int_{0}^{t} g(s) d s\right) \| u_{m}(t)\right) \|^{2}\right] \\
& \left.+\frac{1}{2} g(t) \| u_{m}(t)\right) \|^{2} \\
& -\frac{1}{2}\left(g^{\prime} \square \nabla u_{m}\right)(t) .
\end{aligned}
$$


De (3.5) y (3.6), obtenemos

$$
\begin{aligned}
\frac{d}{d t} E_{1}(t)= & \left(h(t), u_{m}^{\prime}(t)\right)+\frac{1}{2} \mu^{\prime}(t)\left\|u_{m}(t)\right\|^{2}-\left(\alpha(t) u_{m}^{\prime}(t), u_{m}^{\prime}(t)\right) \\
& \left.-\frac{1}{2} g(t) \| u_{m}(t)\right) \|^{2}+\frac{1}{2}\left(g^{\prime} \square \nabla u_{m}\right)(t) .
\end{aligned}
$$

donde

$$
\begin{aligned}
E_{1}(t):= & \frac{1}{2}\left|u_{m}^{\prime}(t)\right|_{2}^{2}+\frac{1}{2} \mu_{g}(t)\left\|u_{m}(t)\right\|^{2} \\
& +\frac{1}{2}\left(g \square \nabla u_{m}\right)(t)+\int_{0}^{t} \beta(s)\left\|u_{m}^{\prime}(s)\right\|^{2} d s \\
\mu_{g}(t):=\mu(t)- & \int_{0}^{t} g(s) d s .
\end{aligned}
$$

De (3.7), se tiene

$$
\frac{d}{d t} E_{1}(t) \leq|h(t)|_{2}\left|u_{m}^{\prime}(t)\right|_{2}+\frac{1}{2}\left|\mu^{\prime}(t)\right|\left\|u_{m}(t)\right\|^{2}+|\alpha(t)|_{L^{\infty}}\left|u_{m}^{\prime}(t)\right|_{2}^{2} .
$$

De (3.9), se obtiene

$$
\frac{d}{d t} E_{1}^{\frac{1}{2}}(t) \leq|h(t)|_{2}+\frac{1}{2}\left[\frac{\left|\mu^{\prime}(t)\right|}{\mu_{g}(t)}+|\alpha(t)|_{L^{\infty}}\right] E_{1}^{\frac{1}{2}}(t) .
$$

Integrando (3.10) y por la desigualdad de Gronwall, resulta

$$
E_{1}(t) \leq\left[E_{1}^{\frac{1}{2}}(0)+\int_{0}^{t}|h(s)|_{2} d s\right]^{2} \exp \left(\int_{0}^{t}\left[\frac{\left|\mu^{\prime}(s)\right|}{\mu_{g}(s)}+|\alpha(s)|_{L^{\infty}}\right] d s\right)
$$

donde

$$
E_{1}(0):=\frac{1}{2}\left|u_{1 m}\right|_{2}^{2}+\frac{1}{2} \mu(0)\left\|u_{0 m}\right\|^{2} .
$$

De (3.11), se tiene

$$
\left|u_{m}^{\prime}(t)\right|_{2}^{2}+\left\|u_{m}(t)\right\|^{2}+\int_{0}^{t}\left\|u_{m}^{\prime}(s)\right\|^{2} d s \leq K_{1}, \forall t \in[0, T]
$$

donde $K_{1}$ es una constante positiva independiente de $m$. 
Estimativa a Priori II. Tomando $w=u_{m}^{\prime \prime}(t)$ en (3.3), obtenemos

$$
\begin{aligned}
\left|u_{m}^{\prime \prime}(t)\right|_{2}^{2}+(\alpha(t) & \left.u_{m}^{\prime}(t), u_{m}^{\prime \prime}(t)\right)+\frac{1}{2} \beta(t) \frac{d}{d t}\left\|u_{m}^{\prime}(t)\right\|^{2} \\
+\mu(t) \frac{d}{d t} & \left(\left(u_{m}(t), u_{m}^{\prime}(t)\right)\right)-\mu(t)\left\|u_{m}^{\prime}(t)\right\|^{2} \\
& \quad-\int_{0}^{t} g(t-s)\left(\left(u_{m}(s), u_{m}^{\prime \prime}(t)\right)\right) d s=\left(h(t), u_{m}^{\prime \prime}(t)\right) .
\end{aligned}
$$

Por diferenciación de la integal $\int_{0}^{t} g(t-s)\left(\left(u_{m}(s), u_{m}^{\prime}(t)\right)\right) d s$, tenemos

$$
\begin{aligned}
-\int_{0}^{t} g(t-s)\left(\left(u_{m}(s), u_{m}^{\prime \prime}(t)\right)\right) d s= & -\frac{d}{d t}\left[\int_{0}^{t} g(t-s)\left(\left(u_{m}(s), u_{m}^{\prime}(t)\right)\right) d s\right] \\
& +\int_{0}^{t} g^{\prime}(t-s)\left(\left(u_{m}(s), u_{m}^{\prime}(t)\right)\right) d s \\
& +g(0)\left(\left(u_{m}(t), \dot{u}_{m}^{\prime}(t)\right)\right) .
\end{aligned}
$$

De (3.13), usando (3.14), obtenemos

$$
\begin{aligned}
\left|u_{m}^{\prime \prime}(t)\right|_{2}^{2} & +\frac{d}{d t}\left[\frac{1}{2} \beta(t)\left\|u_{m}^{\prime}(t)\right\|^{2}+\mu(t)\left(\left(u_{m}(t), u_{m}^{\prime}(t)\right)\right)\right. \\
-\int_{0}^{t} g( & \left.t-s)\left(\left(u_{m}(s), u_{m}^{\prime}(t)\right)\right) d s\right]=\left(h(t), u_{m}^{\prime \prime}(t)\right) \\
& +\frac{1}{2} \beta^{\prime}(t)\left\|u_{m}^{\prime}(t)\right\|^{2}+\mu^{\prime}(t)\left(\left(u_{m}(t), u_{m}^{\prime}(t)\right)\right) \\
& -\left(\alpha(t) u_{m}^{\prime}(t), u_{m}^{\prime \prime}(t)\right)+\mu(t)\left\|u_{m}^{\prime}(t)\right\|^{2} \\
& -g(0)\left(\left(u_{m}(t), u_{m}^{\prime}(t)\right)\right)-\int_{0}^{t} g^{\prime}(t-s)\left(\left(u_{m}(s), u_{m}^{\prime}(t)\right)\right) d s
\end{aligned}
$$

Por (H4), utilizando la desigualdad $a b \leq \delta a^{2}+\frac{1}{4 \delta} b^{2}, \forall a, b \geq 0, \forall \delta>0$ y la desigualdad de Hölder, obtenemos

$$
\begin{aligned}
&-\int_{0}^{t} g^{\prime}(t-s)\left(\left(u_{m}(s), u_{m}^{\prime}(t)\right)\right) d s \leq \eta\left\|u_{m}^{\prime}(t)\right\|^{2} \\
&+\frac{C_{1}^{2}}{4 \eta}|g|_{L^{1}}\left(g *\left\|u_{m}\right\|^{2}\right)(t), \\
&-g(0)\left(\left(u_{m}(t), u_{m}^{\prime}(t)\right)\right) \leq \eta\left\|u_{m}^{\prime}(t)\right\|^{2}+\frac{g^{2}(0)}{4 \eta}\left\|u_{m}(t)\right\|^{2} \\
& \mu^{\prime}(t)\left(\left(u_{m}(t), u_{m}^{\prime}(t)\right)\right) \leq \eta\left\|u_{m}^{\prime}(t)\right\|^{2}+\frac{1}{4 \eta}\left|\mu^{\prime}(t)\right|^{2}\left\|u_{m}(t)\right\|^{2}
\end{aligned}
$$




$$
\begin{gathered}
-\left(\alpha(t) u_{m}^{\prime}(t), u_{m}^{\prime \prime}(t)\right) \leq \varepsilon\left|u_{m}^{\prime \prime}(t)\right|_{2}^{2}+\frac{1}{4 \varepsilon}|\alpha(t)|_{L^{\infty}}^{2}\left|u_{m}^{\prime}(t)\right|_{2}^{2}, \\
\left(h(t), u_{m}^{\prime \prime}(t)\right) \leq \varepsilon\left|u_{m}^{\prime \prime}(t)\right|_{2}^{2}+\frac{1}{4 \varepsilon}|h(t)|_{2}^{2}, \\
\int_{0}^{t} g(t-s)\left(\left(u_{m}(s), u_{m}^{\prime}(t)\right)\right) d s \leq \eta\left\|u_{m}^{\prime}(t)\right\|^{2} \\
+\frac{1}{4 \eta}|g|_{L^{1}} \int_{0}^{t} g(t-s)\left\|u_{m}(s)\right\|^{2} d s
\end{gathered}
$$

donde $0<\varepsilon \leq \frac{1}{4}$ y $0<\eta \leq \frac{1}{4} \beta_{0}$ son constantes.

Integrando (3.15), usando (3.16)-(3.20), la desigualdad de Young para la convolución y (3.21), obtenemos

$$
\begin{aligned}
(1-2 \varepsilon) \int_{0}^{t}\left|u_{m}^{\prime \prime}(s)\right|_{2}^{2} d s & +\left(\frac{1}{2} \beta(t)-2 \eta\right)\left\|u_{m}^{\prime}(t)\right\|^{2} \\
\leq & \frac{1}{2} \beta(0)\left\|u_{1 m}\right\|^{2}+\mu(0)\left\|u_{0 m}\right\|\left\|u_{1 m}\right\| \\
& +\int_{0}^{t}\left[3 \eta+\frac{1}{2}\left|\beta^{\prime}(s)\right|+\mu(s)\right]\left\|u_{m}^{\prime}(s)\right\|^{2} d s \\
& +\frac{1}{4 \eta} \int_{0}^{t}\left[g^{2}(0)+\left|\mu^{\prime}(s)\right|^{2}+C_{1}^{2}|g|_{L^{1}}^{2}\right. \\
& \left.+|g|_{L^{1}} g(s)\right]\left\|u_{m}(s)\right\|^{2} d s \\
& +\frac{1}{4 \varepsilon} \int_{0}^{t}|\alpha(s)|_{L^{\infty}}^{2}\left|u_{m}^{\prime}(s)\right|_{2}^{2} d s \\
& +\frac{1}{4 \varepsilon} \int_{0}^{t}|h(s)|_{2}^{2} d s+\frac{1}{4 \eta}|\mu(t)|^{2}\left\|u_{m}(t)\right\|^{2} .
\end{aligned}
$$

De (3.22), escogiendo $\varepsilon=\frac{1}{4}$ y $\eta=\frac{1}{8} \beta_{0}$, y utilizando la estimativa (3.12), se tiene

$$
\begin{aligned}
& \frac{1}{4} \int_{0}^{t}\left|u_{m}^{\prime \prime}(s)\right|_{2}^{2} d s+\frac{1}{4} \beta_{0}\left\|u_{m}^{\prime}(t)\right\|^{2} \\
& \leq \frac{1}{2} \beta(0)\left\|u_{1 m}\right\|^{2}+\mu(0)\left\|u_{0 m}\right\|\left\|u_{1 m}\right\|+\frac{2}{\beta_{0}} K_{1} \mu_{1}^{2} \\
&+\int_{0}^{t}\left[|h(s)|_{2}^{2}+K_{1}|\alpha(s)|_{L^{\infty}}^{2}+\frac{2 K_{1}}{\beta_{0}}\left(g^{2}(0)\right.\right. \\
&\left.\left.+\left|\mu^{\prime}(s)\right|^{2}+C_{1}^{2}|g|_{L^{1}}^{2}+|g|_{L^{1}} g(s)\right)\right] d s \\
&+\int_{0}^{t}\left[\frac{3}{8} \beta_{0}+\frac{1}{2}\left|\beta^{\prime}(s)\right|+\mu(s)\right]\left\|u_{m}^{\prime}(s)\right\|^{2} d s
\end{aligned}
$$

donde $\mu_{1}:=\sup _{t \in[0, T]} \mu(t)$. 
De (3.23) por la desigualdad de Gronwall, tenemos

$$
\left\|u_{m}^{\prime}(t)\right\|^{2}+\int_{0}^{t}\left|u_{m}^{\prime \prime}(s)\right|_{2}^{2} d s \leq K_{2}, \forall t \in[0, T],
$$

donde $K_{2}$ es una constante positiva independiente de $m$.

Estimativa a Priori III. Tomando $w=-\Delta u_{m}(t)$ en (3.3), obtenemos

$$
\begin{aligned}
\frac{d}{d t}\left[\frac{1}{2} \beta(t)\left|\Delta u_{m}(t)\right|_{2}^{2}-\right. & \left.\left(u_{m}^{\prime}(t), \Delta u_{m}(t)\right)\right]+\mu(t)\left|\Delta u_{m}(t)\right|_{2}^{2} \\
=( & \left.h(t),-\Delta u_{m}(t)\right)+\left\|u_{m}^{\prime}(t)\right\|^{2} \\
& +\left(\alpha(t) u_{m}^{\prime}(t), \Delta u_{m}(t)\right)+\frac{1}{2} \beta^{\prime}(t)\left|\Delta u_{m}(t)\right|_{2}^{2} \\
& +\int_{0}^{t} g(t-s)\left(\Delta u_{m}(s), \Delta u_{m}(t)\right) d s .
\end{aligned}
$$

Por la desigualdad de Hölder, resulta

$$
\begin{aligned}
\int_{0}^{t} g(t-s)\left(\Delta u_{m}(s), \Delta u_{m}(t)\right) d s \leq & \eta\left|\Delta u_{m}(t)\right|_{2}^{2} \\
& +\frac{1}{4 \eta}|g|_{L^{1}} \int_{0}^{t} g(t-s)\left|\Delta u_{m}(s)\right|_{2}^{2} d s,
\end{aligned}
$$

donde $0<\eta<\frac{1}{2} m_{0}$ constante. De (3.25) y por (3.26), se tiene

$$
\begin{aligned}
\frac{d}{d t}\left[\frac{1}{2} \beta(t) \mid \Delta\right. & \left.\left.u_{m}(t)\right|_{2} ^{2}-\left(u_{m}^{\prime}(t), \Delta u_{m}(t)\right)\right]+(\mu(t)-\eta)\left|\Delta u_{m}(t)\right|_{2}^{2} \\
\leq & |h(t)|_{2}\left|\Delta u_{m}(t)\right|_{2}+\left\|u_{m}^{\prime}(t)\right\|^{2} \\
& +|\alpha(t)|_{L^{\infty}}\left|u_{m}^{\prime}(t)\right|_{2}\left|\Delta u_{m}(t)\right|_{2}+\frac{1}{2}\left|\beta^{\prime}(t)\right|\left|\Delta u_{m}(t)\right|_{2}^{2} \\
& +\frac{1}{4 \eta}|g|_{L^{1}} \int_{0}^{t} g(t-s)\left|\Delta u_{m}(s)\right|_{2}^{2} d s .
\end{aligned}
$$

Integrando (3.27), usando las estimativas (3.12) y (3.24), la desigualdad de Young para la convolución y utilizando la desigualdad $a b \leq \delta a^{2}+\frac{1}{4 \delta} b^{2}, \forall a, b \geq 0, \forall \delta>0$, obtenemos

$$
\begin{aligned}
\left(\frac{1}{2} \beta(t)-\lambda\right)\left|\Delta u_{m}(t)\right|_{2}^{2} & +\int_{0}^{t}(\mu(s)-\eta)\left|\Delta u_{m}(s)\right|_{2}^{2} d s \\
\leq & \frac{1}{2} \beta(0)\left|\Delta u_{0 m}\right|_{2}^{2}+\left|u_{1 m}\right|_{2}\left|\Delta u_{0 m}\right|_{2}+\frac{K_{1}}{4 \lambda} \\
& +\int_{0}^{t}\left[K_{2}+\frac{1}{4 \eta}|h(s)|_{2}^{2}+\frac{K_{1}^{2}}{4 \eta}|\alpha(s)|_{L^{\infty}}^{2}\right] d s \\
& +\int_{0}^{t}\left[\frac{1}{2}\left|\beta^{\prime}(s)\right|+\frac{1}{4 \eta}|g|_{L^{1}}^{2}\right]\left|\Delta u_{m}(s)\right|_{2}^{2} d s
\end{aligned}
$$


donde $0<\lambda<\frac{1}{2} \beta_{0}$ constante.

De (3.28), escogiendo $\lambda=\frac{1}{4} \beta_{0}$ y $\eta=\frac{1}{4} m_{0}$, resulta

$$
\begin{aligned}
\frac{1}{4} \beta_{0}\left|\Delta u_{m}(t)\right|_{2}^{2}+\frac{1}{4} m_{0} & \int_{0}^{t}\left|\Delta u_{m}(s)\right|_{2}^{2} d s \\
\leq & \frac{1}{2} \beta(0)\left|\Delta u_{0 m}\right|_{2}^{2}+\left|u_{1 m}\right|_{2}\left|\Delta u_{0 m}\right|_{2}+\frac{K_{1}}{\beta_{0}} \\
& +\int_{0}^{t}\left[K_{2}+\frac{1}{m_{0}}|h(s)|_{2}^{2}+\frac{K_{1}^{2}}{m_{0}}|\alpha(s)|_{L^{\infty}}^{2}\right] d s \\
& +\int_{0}^{t}\left[\frac{1}{2}\left|\beta^{\prime}(s)\right|+\frac{1}{m_{0}}|g|_{L^{1}}^{2}\right]\left|\Delta u_{m}(s)\right|_{2}^{2} d s .
\end{aligned}
$$

De (3.29) por la desigualdad de Gronwall, tenemos

$$
\left|\Delta u_{m}(t)\right|_{2}^{2}+\int_{0}^{t}\left|\Delta u_{m}(s)\right|_{2}^{2} d s \leq K_{3}, \forall t \in[0, T],
$$

donde $K_{3}$ es una constante positiva independiente de $m$.

Pasaje al límite. De las estimativas (3.12),(3.24) y (3.30), existen subsucesiones $\left\{u_{\nu}\right\},\left\{u_{\nu}^{\prime}\right\}$ y $\left\{u_{\nu}^{\prime \prime}\right\}$ de $\left\{u_{m}\right\},\left\{u_{m}^{\prime}\right\}$ y $\left\{u_{m}^{\prime \prime}\right\}$, respectivamente, tales que

$$
\begin{array}{lll}
u_{\nu} \stackrel{*}{*} u & \text { en } & L^{\infty}\left(0, T ; H_{0}^{1}(\Omega) \cap H^{2}(\Omega)\right), \\
u_{\nu}^{\prime} \stackrel{*}{\rightarrow} u^{\prime} & \text { en } \quad L^{\infty}\left(0, T ; H_{0}^{1}(\Omega)\right), \\
u_{\nu}^{\prime} \rightarrow u^{\prime} & \text { en } \quad L^{2}\left(0, T ; H_{0}^{1}(\Omega)\right), \\
u_{\nu}^{\prime \prime} \rightarrow u^{\prime \prime} & \text { en } & L^{2}\left(0, T ; L^{2}(\Omega)\right) .
\end{array}
$$

Por (3.31) y de la ecuación en (3.3), por pasaje al límite, resulta

$$
\begin{aligned}
& u_{t t}+\alpha(x, t) u_{t}-\beta(t) \Delta u_{t}-\mu(t) \Delta u \\
& \quad+\int_{0}^{t} g(t-s) \Delta u(s) d s=h(x, t) \quad \text { en } \quad L^{2}\left(0, T ; H^{-1}(\Omega)\right) .
\end{aligned}
$$

Los datos iniciales se verifica de modo estandar. La estimativa (3.2) se obtiene de manera similar que (3.11). La unicidad resulta de la estimativa (3.2). Esto concluye la demostración del Lema 3.1.

Lema 3.2. Supongamos que las funciones $\mu, \alpha, \beta$ y g satisfacen las hipótesis (H1), (H2), (H3) y (H4), respectivamente, y que $u_{0} \in H_{0}^{1}(\Omega), u_{1} \in L^{2}(\Omega)$ y $h \in L^{2}\left(0, T ; L^{2}(\Omega)\right)$. Entonces el problema (3.1) admite una única solución u sobre [0,T] tal que

$$
\begin{aligned}
& u \in C\left([0, T] ; H_{0}^{1}(\Omega)\right), \\
& u^{\prime} \in C\left([0, T] ; L^{2}(\Omega)\right) \cap L^{2}\left(0, T ; H_{0}^{1}(\Omega)\right), \\
& u^{\prime \prime} \in L^{2}\left(0, T ; H^{-1}(\Omega)\right) .
\end{aligned}
$$

Además, la solución u verifica la estimativa (3.2). 
Demostración. Como $H_{0}^{1}(\Omega) \cap H^{2}(\Omega)$ es denso en $H_{0}^{1}(\Omega)$ y $H_{0}^{1}(\Omega)$ es denso en $L^{2}(\Omega)$, entonces existen sucesiones $\left\{u_{0 m}\right\} \subset H_{0}^{1}(\Omega) \cap H^{2}(\Omega),\left\{u_{1 m}\right\} \subset H_{0}^{1}(\Omega)$ tales que

$$
\begin{aligned}
& u_{0 m} \longrightarrow u_{0} \text { en } \quad H_{0}^{1}(\Omega), \\
& u_{1 m} \longrightarrow u_{1} \text { en } \quad L^{2}(\Omega) .
\end{aligned}
$$

De esta manera, para cada $m \in \mathbb{N}$, consideremos el siguiente problema lineal

$$
\begin{array}{ll}
u_{m}^{\prime \prime}+\alpha(x, t) u_{m}^{\prime}-\beta(t) \Delta u_{m}^{\prime}-\mu(t) \Delta u_{m} & \\
\quad+\int_{0}^{t} g(t-s) \Delta u_{m}(s) d s=h(x, t) & \text { en } \Omega \times] 0, T[, \\
u_{m}=0 & \text { en } \partial \Omega \times] 0, T[, \\
u_{m}(x, 0)=u_{0 m}(x), \quad u_{m}^{\prime}(x, 0)=u_{1 m}(x) & \text { en } \Omega .
\end{array}
$$

Por el Lema 3.1, para cada $m \in \mathbb{N}$, existe una única función $u_{m}$ tal que

$$
\begin{aligned}
& u_{m}^{\prime \prime}+\alpha(x, t) u_{m}^{\prime}-\beta(t) \Delta u_{m}^{\prime}-\mu(t) \Delta u_{m} \\
& +\int_{0}^{t} g(t-s) \Delta u_{m}(s) d s=h(x, t) \text { en } L^{2}\left(0, T ; H^{-1}(\Omega)\right), \\
& \quad u_{m} \in L^{\infty}\left(0, T ; H_{0}^{1}(\Omega) \cap H^{2}(\Omega)\right), \\
& \quad u_{m}^{\prime} \in L^{\infty}\left(0, T ; H_{0}^{1}(\Omega)\right) \cap L^{2}\left(0, T ; H_{0}^{1}(\Omega)\right), \\
& \quad u_{m}^{\prime \prime} \in L^{2}\left(0, T ; L^{2}(\Omega)\right)
\end{aligned}
$$

y verifica la estimativa (3.2).

De (3.36), obtenemos

$$
\begin{aligned}
& u_{m} \in C\left([0, T] ; H_{0}^{1}(\Omega)\right) \\
& u_{m}^{\prime} \in C\left([0, T] ; L^{2}(\Omega)\right) \cap L^{2}\left(0, T ; H_{0}^{1}(\Omega)\right) .
\end{aligned}
$$

Por (3.33), $\left\{u_{0 m}\right\}$ y $\left\{u_{1 m}\right\}$ son sucesiones de Cauchy en $H_{0}^{1}(\Omega)$ y en $L^{2}(\Omega)$, respectivamente. Por la estimativa (3.2), resulta que $\left\{u_{m}\right\}$ y $\left\{u_{m}^{\prime}\right\}$ son sucesiones de Cauchy en $C\left([0, T] ; H_{0}^{1}(\Omega)\right)$ y en $C\left([0, T] ; L^{2}(\Omega)\right) \cap L^{2}\left(0, T ; H_{0}^{1}(\Omega)\right)$, respectivamente. Asi existen $u \in C\left([0, T] ; H_{0}^{1}(\Omega)\right)$ y $u^{\prime} \in C\left([0, T] ; L^{2}(\Omega)\right) \cap L^{2}\left(0, T ; H_{0}^{1}(\Omega)\right)$ tales que

$$
\begin{array}{lll}
u_{m} \longrightarrow u & \text { en } & C\left([0, T] ; H_{0}^{1}(\Omega)\right), \\
u_{m}^{\prime} \longrightarrow u^{\prime} & \text { en } & C\left([0, T] ; L^{2}(\Omega)\right) \cap L^{2}\left(0, T ; H_{0}^{1}(\Omega)\right) .
\end{array}
$$

Por (3.37) y de (3.35), por pasaje al límite, resulta (3.32). De (3.32) se obtiene $u^{\prime \prime} \in$ $L^{2}\left(0, T ; H^{-1}(\Omega)\right)$. De aqui el resto es similar al Lema 3.1. Esto concluye la demostración del Lema 3.2.

Lema 3.3. Supongamos que las funciones $\mu, \alpha, \beta$ y g satisfacen las hipótesis (H1), (H2), (H3) y (H4), respectivamente, y que $u_{0} \in H_{0}^{1}(\Omega) \cap H^{2}(\Omega), u_{1} \in L^{2}(\Omega)$ y $h \in L^{2}\left(0, T ; L^{2}(\Omega)\right)$. Entonces el problema (3.1) admite una única solución u sobre $[0, T]$ tal que

$$
\begin{aligned}
& u \in C\left([0, T] ; H_{0}^{1}(\Omega) \cap H^{2}(\Omega)\right), \\
& u^{\prime} \in C\left([0, T] ; L^{2}(\Omega)\right) \cap L^{2}\left(0, T ; H_{0}^{1}(\Omega)\right), \\
& u^{\prime \prime} \in L^{2}\left(0, T ; H^{-1}(\Omega)\right) .
\end{aligned}
$$


Además, la solución u verifica la siguiente estimativa

$$
E_{2}(t) \leq\left[E_{0}^{\frac{1}{2}}+2 \int_{0}^{t}|h(s)|_{2} d s\right]^{2} \exp \left(\int_{0}^{t} F(s) d s\right), \forall t \in[0, T]
$$

donde

$$
\begin{aligned}
& E_{2}(t):=\frac{1}{10}\left|u^{\prime}(t)\right|_{2}^{2}+\frac{1}{10} \beta_{0}^{2}|\Delta u(t)|_{2}^{2}+\frac{1}{2} \mu_{g}(t)\|u(t)\|^{2}+\frac{1}{2}(g \square \nabla u)(t), \\
& \mu_{g}(t):=\mu(t)-\int_{0}^{t} g(s) d s, \\
& E_{0}:=\frac{9}{10}\left|u_{1}\right|_{2}^{2}+\frac{3}{10} \beta^{2}(0)\left|\Delta u_{0}\right|_{2}^{2}+\frac{1}{2} \mu(0)\left\|u_{0}\right\|^{2}, \\
& F(t):=\frac{\left|\beta^{\prime}(t)\right|}{\beta(t)}+\frac{\left|\mu^{\prime}(t)\right|}{\mu_{g}(t)}+4|\alpha(t)|_{L^{\infty}} .
\end{aligned}
$$

Demostración. La primera parte es similar a la prueba del Lema 3.2. Probemos la estimativa $(3,38)$.

Multiplicando la ecuación en $(3,1)$ por $u^{\prime}$, integrando sobre $\Omega$ y procediendo como para $(3,9)$, se obtiene

$$
\begin{aligned}
\frac{d}{d t}\left[\frac{1}{2}\left|u^{\prime}(t)\right|_{2}^{2}+\frac{1}{2} \mu_{g}(t) \|\right. & \left.\|(t)\|^{2}+\frac{1}{2}(g \square \nabla u)(t)\right] \\
+\beta(t)\left\|u^{\prime}(t)\right\|^{2} & \leq|h(t)|_{2}\left|u^{\prime}(t)\right|_{2} \\
& +\frac{1}{2}\left|\mu^{\prime}(t)\right|\|u(t)\|^{2}+|\alpha(t)|_{L^{\infty}}\left|u^{\prime}(t)\right|_{2}^{2} .
\end{aligned}
$$

También multiplicando la ecuación en (3.1) por $-\Delta u$, integrando sobre $\Omega$ y procediendo como para (3.27), se obtiene

$$
\begin{aligned}
\frac{d}{d t}\left[\frac{1}{2} \beta(t)|\Delta u(t)|_{2}^{2}\right. & \left.-\left(u^{\prime}(t), \Delta u(t)\right)\right]+(\mu(t)-\eta)|\Delta u(t)|_{2}^{2} \\
\leq & |h(t)|_{2}|\Delta u(t)|_{2}+\left\|u^{\prime}(t)\right\|^{2} \\
& +|\alpha(t)|_{L^{\infty}}\left|u^{\prime}(t)\right|_{2}|\Delta u(t)|_{2}+\frac{1}{2}\left|\beta^{\prime}(t)\right||\Delta u(t)|_{2}^{2} \\
& +\frac{1}{4 \eta}|g|_{L^{1}} \int_{0}^{t} g(t-s)|\Delta u(s)|_{2}^{2} d s
\end{aligned}
$$

donde $0<\eta \leq \frac{1}{2}|g|_{L^{1}}$.

Multiplicamos (3.39) por $\lambda, 0<\lambda<2 \beta_{0}$, (3.40) por $\varepsilon, 0<\varepsilon<2 \beta_{0}^{2}$ y sumando los 
resultados, se obtiene

$$
\begin{aligned}
& \beta_{0} \frac{d}{d t} E(t)+(\lambda \beta(t)-\varepsilon)\left\|u^{\prime}(t)\right\|^{2}+\varepsilon(\mu(t)-\eta)|\Delta u(t)|_{2}^{2} \\
& \leq \mid\left.h(t)\right|_{2}\left[\lambda\left|u^{\prime}(t)\right|_{2}+\varepsilon|\Delta u(t)|_{2}\right] \\
&+|\alpha(t)|_{L^{\infty}}\left[\lambda\left|u^{\prime}(t)\right|_{2}^{2}+\varepsilon\left|u^{\prime}(t)\right|_{2}|\Delta u(t)|_{2}\right] \\
&+\frac{1}{2} \lambda\left|\mu^{\prime}(t)\right|\|u(t)\|^{2}+\frac{1}{2} \varepsilon\left|\beta^{\prime}(t)\right||\Delta u(t)|_{2}^{2} \\
&+\frac{\varepsilon}{4 \eta}|g|_{L^{1}} \int_{0}^{t} g(t-s)|\Delta u(s)|_{2}^{2} d s
\end{aligned}
$$

donde

$$
\begin{aligned}
\beta_{0} E(t):=\frac{1}{2} \lambda & {\left[\left|u^{\prime}(t)\right|_{2}^{2}+\mu_{g}(t)\|u(t)\|^{2}+(g \square \nabla u)(t)\right] } \\
& +\frac{1}{2} \varepsilon\left[\beta(t)|\Delta u(t)|_{2}^{2}-2\left(u^{\prime}(t), \Delta u(t)\right)\right] .
\end{aligned}
$$

De (3.42), utilizando $\left|\varepsilon\left(u^{\prime}(t), \Delta u(t)\right)\right| \leq \frac{\varepsilon}{\lambda}\left|u^{\prime}(t)\right|_{2}^{2}+\frac{\varepsilon \lambda}{4}|\Delta u(t)|_{2}^{2}$, resulta

$$
\begin{aligned}
\beta_{0} E(t) \geq & \left(\frac{1}{2} \lambda-\frac{\varepsilon}{\lambda}\right)\left|u^{\prime}(t)\right|_{2}^{2}+\varepsilon\left(\frac{1}{2} \beta_{0}-\frac{\lambda}{4}\right)|\Delta u(t)|_{2}^{2} \\
& +\frac{1}{2} \lambda \mu_{g}(t)\|u(t)\|^{2}+\frac{1}{2} \lambda(g \square \nabla u)(t) .
\end{aligned}
$$

También, se tiene

$$
\begin{aligned}
\beta_{0} E(t) \leq & \left(\frac{1}{2} \lambda+\frac{\varepsilon}{\lambda}\right)\left|u^{\prime}(t)\right|_{2}^{2}+\varepsilon\left(\frac{1}{2} \beta(t)+\frac{\lambda}{4}\right)|\Delta u(t)|_{2}^{2} \\
& +\frac{1}{2} \lambda \mu_{g}(t)\|u(t)\|^{2}+\frac{1}{2} \lambda(g \square \nabla u)(t) .
\end{aligned}
$$

De (3.43) y (3.44), escogiendo $\lambda=\beta_{0}$ y $\varepsilon=\frac{2}{5} \beta_{0}^{2}$, resulta

$$
\begin{gathered}
E(t) \geq E_{2}(t), \forall t \in[0, T], \\
E(0) \leq E_{0},
\end{gathered}
$$

donde

$$
\begin{aligned}
& E_{2}(t):=\frac{1}{10}\left|u^{\prime}(t)\right|_{2}^{2}+\frac{1}{10} \beta_{0}^{2}|\Delta u(t)|_{2}^{2}+\frac{1}{2} \mu_{g}(t)\|u(t)\|^{2}+\frac{1}{2}(g \square \nabla u)(t), \\
& E_{0}:=\frac{9}{10}\left|u_{1}\right|_{2}^{2}+\frac{3}{10} \beta^{2}(0)\left|\Delta u_{0}\right|_{2}^{2}+\frac{1}{2} \mu(0)\left\|u_{0}\right\|^{2} .
\end{aligned}
$$


De (3.41), se obtiene

$$
\begin{aligned}
\frac{1}{2} \frac{d}{d t} E(t)+\frac{3}{10} \beta_{0}\left\|u^{\prime}(t)\right\|^{2} & +\frac{1}{5} \beta_{0}\left(m_{0}-\eta\right)|\Delta u(t)|_{2}^{2} \\
\leq & |h(t)|_{2}\left[E^{\frac{1}{2}}(t)+E^{\frac{1}{2}}(t)\right] \\
& +|\alpha(t)|_{L^{\infty}}[E(t)+E(t)] \\
& +\frac{1}{2} \frac{\left|\mu^{\prime}(t)\right|}{\mu_{g}(t)} E(t)+\frac{1}{2} \frac{\left|\beta^{\prime}(t)\right|}{\beta(t)} E(t) \\
& +\frac{\beta_{0}}{20 \eta}|g|_{L^{1}} \int_{0}^{t} g(t-s)|\Delta u(s)|_{2}^{2} d s .
\end{aligned}
$$

Integrando (3.47) y usando la desigualdad de Young para la convolución, obtenemos

$$
\begin{aligned}
& \frac{1}{2} E(t)+ \frac{3}{10} \beta_{0} \int_{0}^{t}\left\|u^{\prime}(s)\right\|^{2} d s \\
&+\frac{1}{5} \beta_{0}\left(m_{0}-\eta-\frac{1}{4 \eta}|g|_{L^{1}}^{2}\right) \int_{0}^{t}|\Delta u(s)|_{2}^{2} d s \\
& \leq \frac{1}{2} E(0)+2 \int_{0}^{t}|h(s)|_{2} E^{\frac{1}{2}}(s) d s \\
& \quad+\int_{0}^{t}\left[\frac{1}{2} \frac{\left|\beta^{\prime}(s)\right|}{\beta(s)}+\frac{1}{2} \frac{\left|\mu^{\prime}(s)\right|}{\mu_{g}(s)}+2|\alpha(s)|_{L^{\infty}}\right] E(s) d s .
\end{aligned}
$$

De (3.48), escogiendo $\eta=\frac{1}{2}|g|_{L^{1}}$, resulta

$$
\begin{aligned}
\frac{1}{2} E(t) \leq & \frac{1}{2} E(0)+2 \int_{0}^{t}|h(s)|_{2} E^{\frac{1}{2}}(s) d s \\
& +\int_{0}^{t} \frac{1}{2}\left[\frac{\left|\beta^{\prime}(s)\right|}{\beta(s)}+\frac{\left|\mu^{\prime}(s)\right|}{\mu_{g}(s)}+4|\alpha(s)|_{L^{\infty}}\right] E(s) d s .
\end{aligned}
$$

De (3.49), por la desigualdad de Gronwall y luego por (3.45)-( 3.46), resulta la estimativa $(3,38)$. Con esto se concluye la demostración del Lema 3.3 .

\section{Existencia y Unicidad}

El objetivo central del presente trabajo es discutir la existencia de soluciones del problema ( 1.1), usando argumentos del Teorema de punto fijo de Banach. Para esto emplearemos resultados del Lema 3.3. 
Definamos el siguiente espacio de dos parámetros, llamado conjunto de soluciones o conjunto admisible

$$
\begin{aligned}
G_{T_{0}, R_{0}}:=\{v & \in C\left(\left[0, T_{0}\right] ; H_{0}^{1}(\Omega) \cap H^{2}(\Omega)\right) ; \\
& v^{\prime} \in C\left(\left[0, T_{0}\right] ; L^{2}(\Omega)\right) \cap L^{2}\left(0, T_{0} ; H_{0}^{1}(\Omega)\right), \\
& \left|v^{\prime}(t)\right|_{2}^{2}+|\Delta v(t)|_{2}^{2} \leq R_{0}^{2}, t \in\left[0, T_{0}\right] \\
& \left.\operatorname{con} v(0)=u_{0} \mathrm{y} v^{\prime}(0)=u_{1}\right\}
\end{aligned}
$$

donde $T_{0}>0$ y $R_{0}>0$. Entonces $G_{T_{0}, R_{0}}$ es un espacio métrico completo con la distancia

$$
d(u, v):=\sup _{0 \leq t \leq T_{0}}\left[\left|u^{\prime}(t)-v^{\prime}(t)\right|_{2}^{2}+|\Delta u(t)-\Delta v(t)|_{2}^{2}\right]^{\frac{1}{2}}
$$

Lema 4.1. Supongamos que la función $M$ satisface la hipótesis (H5). Si $u, v \in G_{T_{0}, R_{0}} y$ $\mu(t ; v):=M\left(\|v(t)\|^{2}\right)$, entonces $\mu \in C\left(\left[0, T_{0}\right]\right), \mu^{\prime} \in L^{\infty}\left(0, T_{0}\right) y$

$$
\begin{aligned}
& |\mu(t ; v)-\mu(s ; v)| \leq 2 M_{1} R_{0}^{2}|t-s|, \forall t \in\left[0, T_{0}\right], \\
& |\mu(t ; u)-\mu(t ; v)| \leq 2 M_{1} B_{1}^{2} R_{0}|\Delta u(t)-\Delta v(t)|_{2}, \forall t \in\left[0, T_{0}\right], \\
& \left|\mu^{\prime}(t ; v)\right| \leq 2 M_{1} R_{0}^{2}, \forall t \in\left[0, T_{0}\right] \\
& 0<m_{0} \leq \mu(t ; v) \leq M_{0}, \forall t \in\left[0, T_{0}\right],
\end{aligned}
$$

donde

$$
\begin{aligned}
& M_{0}:=\sup \left\{M(s) ; 0 \leq s \leq B_{1}^{2} R_{0}^{2}\right\} \\
& M_{1}:=\sup \left\{\left|M^{\prime}(s)\right| ; 0 \leq s \leq B_{1}^{2} R_{0}^{2}\right\}
\end{aligned}
$$

\section{Demostración.}

Por el Teorema de valor medio y la desigualdad de Sobolev-Poincaré, se obtienen los resultados.

Lema 4.2. Supongamos que la función $f$ satisface la hipótesis (H6). Si $u, v \in G_{T_{0}, R_{0}}$ y $h(t ; v):=f(v(t))$, entonces $h \in C\left(\left[0, T_{0}\right] ; L^{2}(\Omega)\right) y$

$$
\begin{aligned}
& |h(t ; v)-h(s ; v)|_{2} \leq 2 K B_{1}^{2(p-1)} R_{0}^{p-2}|\Delta v(t)-\Delta v(s)|_{2}, \forall t \in\left[0, T_{0}\right], \\
& |h(t ; u)-h(t ; v)|_{2} \leq 2 K B_{1}^{2(p-1)} R_{0}^{p-2}|\Delta u(t)-\Delta v(t)|_{2}, \forall t \in\left[0, T_{0}\right], \\
& |h(t ; v)|_{2} \leq K B_{1}^{2(p-1)} R_{0}^{p-1}, \forall t \in\left[0, T_{0}\right] .
\end{aligned}
$$

\section{Demostración.}

Por la desigualdad de Sobolev-Poincaré, se obtienen los resultados.

Teorema 4.3 (Existencia Local). Supongamos que las funciones $\alpha, \beta, g, M$ y $f$ satisfacen las hipótesis (H2), (H3), (H4), (H5) y (H6), respectivamente, y que $u_{0} \in H_{0}^{1}(\Omega) \cap H^{2}(\Omega)$ y $u_{1} \in L^{2}(\Omega)$. Entonces existe $T_{0}>0$ y el problema (1.1) admite una única solución u sobre $\left[0, T_{0}\right]$ tal que

$$
\begin{aligned}
& u \in C\left(\left[0, T_{0}\right] ; H_{0}^{1}(\Omega) \cap H^{2}(\Omega)\right), \\
& u^{\prime} \in C\left(\left[0, T_{0}\right] ; L^{2}(\Omega)\right) \cap L^{2}\left(0, T_{0} ; H_{0}^{1}(\Omega)\right), \\
& u^{\prime \prime} \in L^{2}\left(0, T_{0} ; H^{-1}(\Omega)\right) .
\end{aligned}
$$




\section{Demostración.}

Existencia de Soluciones. Para cada $v \in G_{T_{0}, R_{0}}$, consideremos el siguiente problema lineal

$$
\begin{array}{ll}
u_{t t}+\alpha(x, t) u_{t}-\beta(t) \Delta u_{t}-\mu(t ; v) \Delta u & \\
\qquad+\int_{0}^{t} g(t-s) \Delta u(s) d s=h(t ; v) & \text { en } \Omega \times] 0, T_{0}[, \\
\begin{array}{ll}
u=0 & \text { en } \partial \Omega \times] 0, T_{0}[, \\
u(x, 0)=u_{0}(x), u_{t}(x, 0)=u_{1}(x) & \text { en } \Omega,
\end{array}
\end{array}
$$

donde $T_{0}>0$ y $R_{0}>0$ serán determinados más tarde, $\mu(t ; v):=M\left(\|v(t)\|^{2}\right)$ y $h(t ; v):=$ $f(v(t))$. Por los Lemas 4.1 y 4.2 , las funciones $\mu(. ; v)$ y $h(. ; v)$, satisfacen las hipótesis del Lema 3.3, entonces existe una única solución $u$ sobre $\left[0, T_{0}\right]$ del problema (4.2) tal que

$$
\begin{aligned}
& u \in C\left(\left[0, T_{0}\right] ; H_{0}^{1}(\Omega) \cap H^{2}(\Omega)\right), \\
& u^{\prime} \in C\left(\left[0, T_{0}\right] ; L^{2}(\Omega)\right) \cap L^{2}\left(0, T_{0} ; H_{0}^{1}(\Omega)\right), \\
& u^{\prime \prime} \in L^{2}\left(0, T_{0} ; H^{-1}(\Omega)\right) .
\end{aligned}
$$

Además, la solución $u$ verifica la siguiente estimativa

$$
E_{2}(t) \leq\left[E_{0}^{\frac{1}{2}}+2 \int_{0}^{t}|h(s ; v)|_{2} d s\right]^{2} \exp \left(\int_{0}^{t} F(s) d s\right), \forall t \in\left[0, T_{0}\right]
$$

donde

$$
\begin{aligned}
& E_{2}(t):=\frac{1}{10}\left|u^{\prime}(t)\right|_{2}^{2}+\frac{1}{10} \beta_{0}^{2}|\Delta u(t)|_{2}^{2}+\frac{1}{2} \mu_{g}(t ; v)\|u(t)\|^{2}+\frac{1}{2}(g \square \nabla u)(t), \\
& \mu_{g}(t ; v):=\mu(t ; v)-\int_{0}^{t} g(s) d s \\
& E_{0}:=\frac{9}{10}\left|u_{1}\right|_{2}^{2}+\frac{3}{10} \beta^{2}(0)\left|\Delta u_{0}\right|_{2}^{2}+\frac{1}{2} M\left(\left\|u_{0}\right\|^{2}\right)\left\|u_{0}\right\|^{2} \\
& F(t):=\frac{\left|\beta^{\prime}(t)\right|}{\beta(t)}+\frac{\left|\mu^{\prime}(t ; v)\right|}{\mu_{g}(t ; v)}+4|\alpha(t)|_{L^{\infty}} .
\end{aligned}
$$

El siguiente paso será mostrar que $u \in G_{T_{0}, R_{0}}$. Por (4.4) y los Lemas 4.1 y 4.2 , obtenemos

$$
\begin{aligned}
\left|u^{\prime}(t)\right|_{2}^{2}+|\Delta u(t)|_{2}^{2} \leq & \frac{10}{\operatorname{mín}\left\{1, \beta_{0}^{2}\right\}}\left[E_{0}^{\frac{1}{2}}+2 K B_{1}^{2(p-1)} R_{0}^{p-1} T_{0}\right]^{2} \\
& \exp \left(\int_{0}^{T_{0}} F_{0}(s) d s\right)
\end{aligned}
$$

donde $F_{0}(t):=\frac{\left|\beta^{\prime}(t)\right|}{\beta_{0}}+\frac{2 M_{1} R_{0}^{2}}{l}+4|\alpha(t)|_{L^{\infty}}$. Escogemos la constante $R_{0}>0$ que satisfaga la relación

$$
R_{0} \geq\left(\frac{20}{\min \left\{1, \beta_{0}^{2}\right\}}\right)^{\frac{1}{2}}\left[E_{0}^{\frac{1}{2}}+2\right] \text {. }
$$


Tomemos $T_{0}>0$ que satisfaga a las relaciones

$$
K B_{1}^{2(p-1)} R_{0}^{p-1} T_{0} \leq 1 \text { y } \exp \left(\int_{0}^{T_{0}} F_{0}(s) d s\right) \leq 2 .
$$

Entonces de (4.5), resulta

$$
\left|u^{\prime}(t)\right|_{2}^{2}+|\Delta u(t)|_{2}^{2} \leq R_{0}^{2}, \forall t \in\left[0, T_{0}\right]
$$

De aqui, $(4,3)$ y desde que $u$ es solución de (4.2), se tiene que $u \in G_{T_{0}, R_{0}}$.

Por el resultado antes obtenido, definamos la aplicación no lineal $\varphi$ como

$$
\begin{aligned}
\varphi: G_{T_{0}, R_{0}} & \longrightarrow G_{T_{0}, R_{0}} \\
v & \longmapsto \varphi(v)=u
\end{aligned}
$$

donde $u$ es la solución del problema (4.2). Esto significa que $\varphi\left(G_{T_{0}, R_{0}}\right) \subset G_{T_{0}, R_{0}}$.

Ahora probaremos que $\varphi$ es una contracción estricta con respecto a la distancia (4.1), es decir,

$$
d\left(\varphi\left(v_{1}\right), \varphi\left(v_{2}\right)\right) \leq \delta d\left(v_{1}, v_{2}\right), \forall v_{1}, v_{2} \in G_{T_{0}, R_{0}}
$$

para un fijo $\delta, 0<\delta<1$.

Sean $v_{1}, v_{2} \in G_{T_{0}, R_{0}}$, entonces $u_{1}=\varphi\left(v_{1}\right)$ y $u_{2}=\varphi\left(v_{2}\right)$ son soluciones del problema (4.2). Haciendo $w=u_{1}-u_{2}$, se tiene que $w$ es solución del problema lineal

$$
\begin{array}{ll}
w_{t t}+\alpha(x, t) w_{t}-\beta(t) \Delta w_{t}-\mu\left(t ; v_{1}\right) w & \\
\qquad \int_{0}^{t} g(t-s) \Delta w(s) d s=h\left(t ; v_{1}, v_{2}\right) & \text { en } \Omega \times] 0, T_{0}[, \\
w=0 & \text { en } \partial \Omega \times] 0, T_{0}[, \\
w(x, 0)=0, w_{t}(x, 0)=0 & \text { en } \Omega,
\end{array}
$$

donde

$$
\begin{aligned}
& \mu\left(t ; v_{1}\right):=M\left(\left\|v_{1}(t)\right\|^{2}\right) \\
& h\left(t ; v_{1}, v_{2}\right):=\left[M\left(\left\|v_{2}(t)\right\|^{2}\right)-M\left(\left\|v_{1}(t)\right\|^{2}\right)\right] \Delta u_{2}(t)+f\left(v_{1}(t)\right)-f\left(v_{2}(t)\right) .
\end{aligned}
$$

Por los Lemas 4.1 y 4.2, obtenemos

$$
\left|h\left(t ; v_{1}, v_{2}\right)\right|_{2} \leq 2\left[M_{1} B_{1}^{2} R_{0}^{2}+K B_{1}^{2(p-1)} R_{0}^{p-2}\right]\left|\Delta v_{1}(t)-\Delta v_{2}(t)\right|_{2} .
$$

Del Lema 4.1, (4.8) y Lema 3.3, existe una única solución $w$ sobre $\left[0, T_{0}\right]$ del problema ( 4.7) y verifica la estimativa

$$
E_{2}(t) \leq\left[2 \int_{0}^{t}\left|h\left(s ; v_{1}, v_{2}\right)\right|_{2} d s\right]^{2} \exp \left(\int_{0}^{t} F_{0}(s) d s\right), \forall t \in\left[0, T_{0}\right]
$$


donde $F_{0}(t):=\frac{\left|\beta^{\prime}(t)\right|}{\beta_{0}}+\frac{2 M_{1} R_{0}^{2}}{l}+4|\alpha(t)|_{L^{\infty}}$. De $(4,9)$, resulta

$$
\begin{aligned}
\left|w^{\prime}(t)\right|_{2}^{2}+|\Delta w(t)|_{2}^{2} \leq & \frac{160}{\operatorname{mín}\left\{1, \beta_{0}^{2}\right\}}\left[M_{1} B_{1}^{2} R_{0}^{2}+K B_{1}^{2(p-1)} R_{0}^{p-2}\right]^{2} \\
& \exp \left(\int_{0}^{T_{0}} F_{0}(s) d s\right) \\
& {\left[\int_{0}^{T_{0}}\left|\Delta v_{1}(t)-\Delta v_{2}(t)\right|_{2} d t\right]^{2} . }
\end{aligned}
$$

Por (4.6), de (4.10), resulta

$$
d\left(\varphi\left(v_{1}\right), \varphi\left(v_{2}\right)\right) \leq\left[\frac{320}{\min \left\{1, \beta_{0}^{2}\right\}}\right]^{\frac{1}{2}}\left[T_{0} M_{1} B_{1}^{2} R_{0}^{2}+R_{0}^{-1}\right] d\left(v_{1}, v_{2}\right) .
$$

Además de las relaciones de $(4,6)$, tomando $T_{0}>0$ que satisfaga la relación

$$
\delta:=\left[\frac{320}{\min \left\{1, \beta_{0}^{2}\right\}}\right]^{\frac{1}{2}}\left[T_{0} M_{1} B_{1}^{2} R_{0}^{2}+R_{0}^{-1}\right]<1,
$$

se tiene de (4.11) que $\varphi$ es una contracción estricta.

Aplicando el teorema de punto fijo de Banach, existe un único $u \in G_{T_{0}, R_{0}}$ tal que $\varphi(u)=$ $u$. Asi, hemos obtenido la existencia de la solución local del problema (1.1)

Unicidad de Soluciones. Sea $u$ la solución construida en la prueba de existencia, la cual pertenece a $G_{T_{0}, R_{0}}$. Consideremos otra solución $v$ de (1.1) tal que

$$
\begin{aligned}
& v \in C\left(\left[0, T_{1}\right] ; H_{0}^{1}(\Omega) \cap H^{2}(\Omega)\right) \\
& v^{\prime} \in C\left(\left[0, T_{1}\right] ; L^{2}(\Omega)\right) \cap L^{2}\left(0, T_{1} ; H_{0}^{1}(\Omega)\right), \\
& v^{\prime \prime} \in L^{2}\left(0, T_{1} ; H^{-1}(\Omega)\right)
\end{aligned}
$$

con $0<T_{1} \leq T_{0}$. Entonces, la función $w=u-v$ satisface el siguiente problema lineal

$$
\begin{aligned}
& w_{t t}+\alpha(x, t) w_{t}-\beta(t) \Delta w_{t}-\mu(t ; u) \Delta w \\
& \left.+\int_{0}^{t} g(t-s) \Delta w(s) d s=h(t ; u, v) \text { en } \Omega \times\right] 0, T_{1}[, \\
& w=0 \quad \text { en } \partial \Omega \times] 0, T_{1}[\text {, } \\
& w(x, 0)=0, w_{t}(x, 0)=0 \quad \text { en } \Omega,
\end{aligned}
$$

donde

$$
\begin{aligned}
& \mu(t ; u):=M\left(\|u(t)\|^{2}\right), \\
& h(t ; u, v):=\left[M\left(\|v(t)\|^{2}\right)-M\left(\|u(t)\|^{2}\right)\right] \Delta v(t)+f(u(t))-f(v(t)) .
\end{aligned}
$$


Por (4.12), existe una constante $C_{0}>0$ tal que

$$
|\Delta v(t)| \leq C_{0}, \forall t \in\left[0, T_{1}\right] .
$$

Por el teorema de valor medio, desigualdad de Sobolev-Poincaré y (4.14), se obtiene

$$
\begin{aligned}
|h(t ; u, v)|_{2} \leq[ & M_{2} B_{1}^{2}\left(R_{0}+C_{0}\right) C_{0} \\
& \left.+K B_{1}^{2(p-1)}\left(R_{0}^{p-2}+C_{0}^{p-2}\right)\right]|\Delta u(t)-\Delta v(t)|_{2},
\end{aligned}
$$

donde $M_{2}:=\sup \left\{\left|M^{\prime}(s)\right| ; 0 \leq s \leq B_{1}^{2} \operatorname{máx}\left\{R_{0}^{2}, C_{0}^{2}\right\}\right\}$.

Del Lema 4.1, (4.15) y Lema 3.3, existe una única solución $w$ sobre $\left[0, T_{1}\right]$ del problema (4.13) y verifica la estimativa

$$
E_{2}(t) \leq\left[2 \int_{0}^{t}|h(s ; u, v)|_{2} d s\right]^{2} \exp \left(\int_{0}^{t} F_{0}(s) d s\right), \forall t \in\left[0, T_{1}\right]
$$

donde $F_{0}(t):=\frac{\left|\beta^{\prime}(t)\right|}{\beta_{0}}+\frac{2 M_{1} R_{0}^{2}}{l}+4|\alpha(t)|_{L^{\infty}}$. De (4.16) y empleando (4.15), resulta

$$
|\Delta w(t)| \leq \mathrm{const} \int_{0}^{t}|\Delta w(s)| d s, \forall t \in\left[0, T_{1}\right] .
$$

De $(4,17)$ y la desigualdad de Gronwall, se tiene que $|\Delta w(t)|=0, \forall t \in\left[0 . T_{1}\right], 0<T_{1} \leq T_{0}$. Esto implica que $w(t)=0, \forall t \in\left[0, T_{1}\right]$. Con esto hemos concluido la unicidad y por tanto la totalidad de la demostración del Teorema 4.3.

Corolario 4.4. Supongamos que las funciones $\alpha, \beta, g, M$ y $f$ satisfacen las hipótesis (H2), (H3), (H4), (H5) y (H6), respectivamente, y que $u_{0} \in H_{0}^{1}(\Omega) \cap H^{2}(\Omega)$ y $u_{1} \in L^{2}(\Omega)$. Entonces existe un único intervalo $\left[0, T_{\operatorname{máx}}\left[\right.\right.$ con $0<T_{\operatorname{máx}} \leq \infty$ y el problema (1.1) admite una única solución u sobre $\left[0, T_{\text {máx }}[\right.$ tal que

$$
\begin{aligned}
& u \in C\left(\left[0, T_{\text {máx }}\left[H_{0}^{1}(\Omega) \cap H^{2}(\Omega)\right),\right.\right. \\
& u^{\prime} \in C\left(\left[0, T_{\text {máx }}\left[; L^{2}(\Omega)\right) \cap L^{2}\left(0, T_{\text {máx }} ; H_{0}^{1}(\Omega)\right),\right.\right. \\
& u^{\prime \prime} \in L^{2}\left(0, T_{\text {máx }} ; H^{-1}(\Omega)\right) .
\end{aligned}
$$

Demostración. Para los datos iniciales $u_{0} \in H_{0}^{1}(\Omega) \cap H^{2}(\Omega)$ y $u_{1} \in L^{2}(\Omega)$, por el Teorema 4.3 , existe $T_{0}>0$ y el problema $(1,1)$ admite una única solución $u$ sobre $\left[0, T_{0}\right]$ tal que

$$
\begin{aligned}
& u \in C\left(\left[0, T_{0}\right] ; H_{0}^{1}(\Omega) \cap H^{2}(\Omega)\right), \\
& u^{\prime} \in C\left(\left[0, T_{0}\right] ; L^{2}(\Omega)\right) \cap L^{2}\left(0, T_{0} ; H_{0}^{1}(\Omega)\right), \\
& u^{\prime \prime} \in L^{2}\left(0, T_{0} ; H^{-1}(\Omega)\right) .
\end{aligned}
$$

Además por (4.18), existe $u\left(T_{0}\right) \in H_{0}^{1}(\Omega) \cap H^{2}(\Omega)$ y $u^{\prime}\left(T_{0}\right) \in L^{2}(\Omega)$. 
Ahora tomando como datos inciales $u\left(T_{0}\right) \in H_{0}^{1}(\Omega) \cap H^{2}(\Omega) \quad$ y $\quad u^{\prime}\left(T_{0}\right) \in L^{2}(\Omega)$, y nuevamente por el Teorema 4.3, existe $T_{1}>0$ y el problema (1.1) admite una única solución $v$ sobre $\left[0, T_{1}\right]$ tal que

$$
\begin{aligned}
& v \in C\left(\left[0, T_{1}\right] ; H_{0}^{1}(\Omega) \cap H^{2}(\Omega)\right) \\
& v^{\prime} \in C\left(\left[0, T_{1}\right] ; L^{2}(\Omega)\right) \cap L^{2}\left(0, T_{1} ; H_{0}^{1}(\Omega)\right) \\
& v^{\prime \prime} \in L^{2}\left(0, T_{1} ; H^{-1}(\Omega)\right)
\end{aligned}
$$

Definamos la función

$$
w(t):= \begin{cases}u(t) & , \text { si } 0 \leq t \leq T_{0} \\ v\left(t-T_{0}\right) & , \text { si } T_{0} \leq t \leq T_{0}+T_{1} .\end{cases}
$$

Entonces para los datos iniciales $u_{0} \in H_{0}^{1}(\Omega) \cap H^{2}(\Omega)$ y $u_{1} \in L^{2}(\Omega)$, w es una única solución del problema (1.1) sobre $\left[0, T_{0}+T_{1}\right]$ tal que tiene la propiedad (4.18).

Ahora consideremos para los datos iniciales $u_{0} \in H_{0}^{1}(\Omega) \cap H^{2}(\Omega)$ y $u_{1} \in L^{2}(\Omega)$, la familia $\left\{u_{i}\right\}_{i \in I}$ de todas las soluciones del problema (1.1) sobre $\left[0, T_{i}\right]$ tal que tiene la propiedad (4.18). Si $T_{i}<T_{j}$, por la unicidad de soluciones implica que $u_{i}$ y $u_{j}$ coinciden en $\left[0, T_{i}\right]$. Entonces el intervalo $\left[0, T_{\text {máx }}[\right.$ dado por

$$
\left[0, T_{\text {máx }}\left[=\bigcup_{i \in I}\left[0, T_{i}\right]\right.\right.
$$

es el intervalo maximal de existencia de la solución $u$ del problema (1.1). Con esto se concluye la demostración del Corolario 4.4.

\section{Referencias}

[1] ANDRADE D. and MOGNON A., Global solutions for a system of Klein-Gordon equations with memory, Bol. Soc. Paran. Mat. 21 1/2 (2003)

[2] BERRIMI S. and MESSAOUDI S. A., Exponential decay of solutions to a viscoelastic equation with nonlinear localized damping, Electronic Journal of Differential Equations, Vol. 2004 (2004), No. 88, pp.1-10

[3] BERRIMI S. and MESSAOUDI S. A., Existence and decay of solutions of a viscoelastic equation with a nonlinear source, Nonlinear Analysis 64 (2006) 2314-2331.

[4] BRÉZIS H., Análisis funcional, Alianza Editorial, Madrid, (1984)

[5] CAVAlCANTI M. M., DOMingos CAVAlCANTI V. N. and SORIANO J. A., Exponential decay for the solution of semilinear viscoelastic wave equations with localized damping, Electronic Journal of Differential Equations, Vol. 2002 (2002), No. 44, pp.1-14 
[6] CODDINGTON E. A. and LENINSON N., Theory of ordinary differential equations, McGraw-Hill, New York, (1955)

[7] KIRCHHOFF G., Vorlesungen über mechanik, Leipzig, Teubner, (1883)

[8] MESSAOUDI S., Blow-up of positive-initial-energy solutions of a nonlinear viscoelastic hyperbolic equation, J. Math. Anal. Appl. 320 (2006) 902-915

[9] WU S. T., Blow-up of solutions for an integro-differential equation with a nonlinear source, Electronic Journal of Differential Equations, Vol. 2006 (2006), No. 45, pp.1-9

[10] WU S. T. and Tsai L. Y., On global existence and blow-up of solutions for an integrodifferential equation with strong damping, Taiwanese Journal of Mathematic Vol.10, No. 4, pp. 979-1014, June (2006)

[11] ZEIDLER E., Nonlinear functional analysis and its applications I: Fixed-point theorems, Springer-Verlag, New York, (1986) 